By C. A. Sanocki and T. A. Winterstein

Open-File Report 99-472

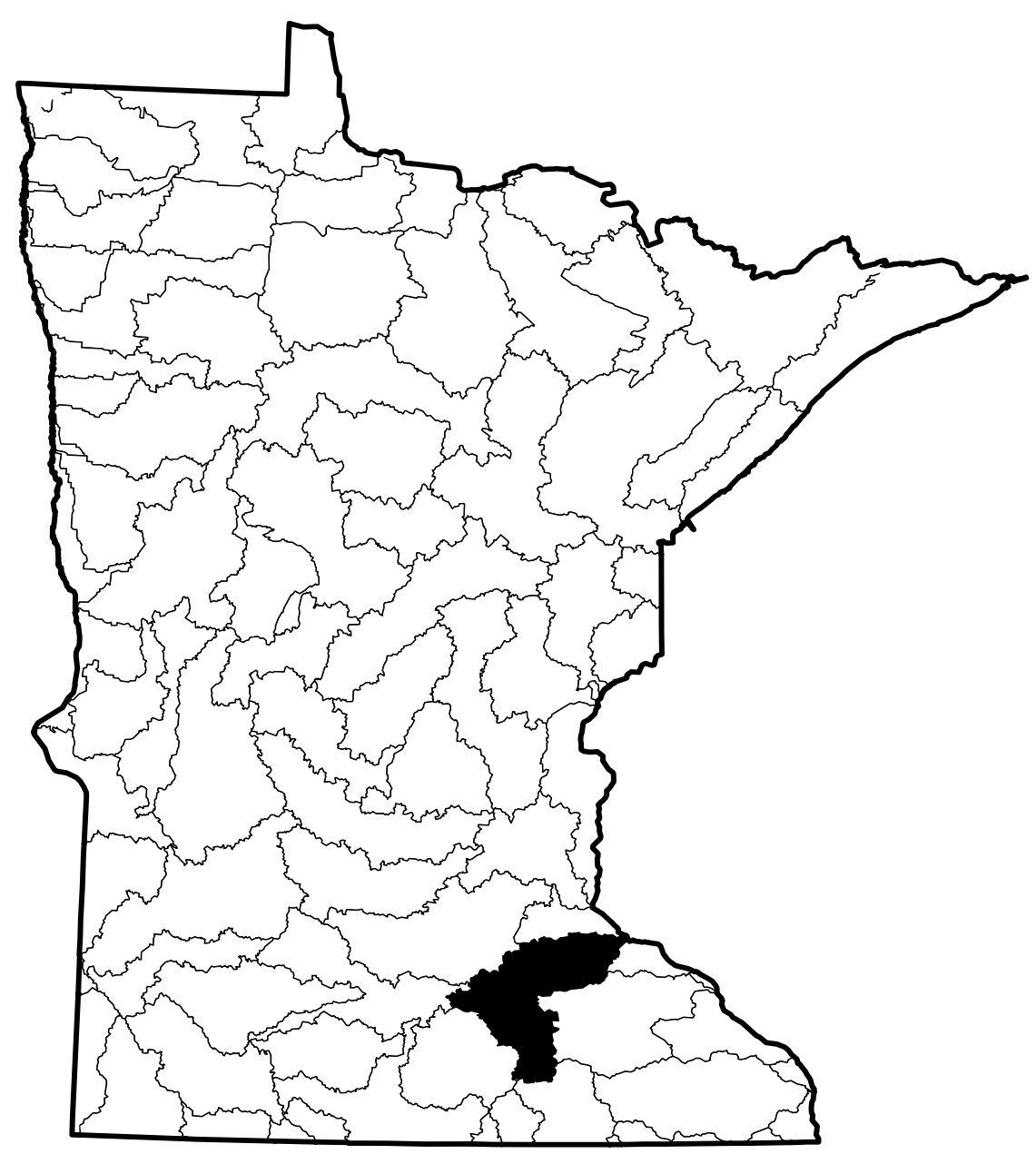

Prepared in cooperation with the

Minnesota Department of Transportation

Mounds View, Minnesota

1999

U.S. Department of the Interior U.S. Geological Survey 


\title{
Physical Characteristics of Stream Subbasins in the Cannon River Basin, Southeastern Minnesota
}

\author{
By C. A. Sanocki and T. A. Winterstein
}

\begin{abstract}
Data that describe the physical characteristics of stream subbasins upstream from selected sites on streams in the Cannon River Basin, located in southeastern Minnesota, are presented in this report. The physical characteristics are the drainage area of the subbasin, the percentage area of the subbasin covered only by lakes, the percentage area of the subbasin covered by both lakes and marsh, the main-channel length, and the main-channel slope. Stream sites include outlets of subbasins of at least 5 square miles, and locations of U.S. Geological Survey high-flow, and continuous-record gaging stations.
\end{abstract}

\section{Introduction}

This is the 15th report in a series detailing subbasin characteristics of streams in Minnesota and adjacent states. The Cannon River Basin drains an area of 1440 square miles and is represented by hydrologic accounting unit 07040002 (U.S. Geological Survey, 1974). The Cannon River Basin includes parts of Blue Earth, Dakota, Freeborn, Goodhue, Le Sueur, Rice, Scott, Steele, Waseca Counties in southeastern Minnesota.

Selected data for sites on streams at outlets of subbasins larger than about 5 square miles; at locations of U.S. Geological Survey (USGS) highflow, and continuous-record gaging stations located in the Cannon River Basin are presented in this report. This report was prepared in cooperation with the Minnesota Department of Transportation.

\section{Methods}

USGS 7-1/2 minute series topographic maps were used as source maps to define subbasin boundaries and to obtain main-channel length, and the contour elevation points used in this report. Paper copies of the maps were used. Lake and marsh data were obtained from U.S. Fish and Wildlife Service National Wetlands Inventory Data (U.S. Fish \& Wildlife Service, 1981-present). A geographic information system (GIS) was used to define the geographic location and extent of the subbasins, lakes, marshes, main-channels, and elevation points. Data digitized from paper copies were in error by no more than twice the horizontal accuracy of National Mapping Standards of 40 feet (Thompson, 1987, p. 104). All thematic (digitized) data were projected into an Albers Equal-Area projection for storage and analysis.

Subbasin boundaries were delineated on the basis of anthropogenic activities and topographic contours. Anthropogenic activities, such as the installation of storm sewers, the drainage of wetlands, and the diversion of streams, may alter the drainage area of a stream; therefore data from field inspections and recent drainage-ditch maps were transferred to the topographic maps. The subbasin boundaries were digitized by the Minnesota Department of Natural Resources (DNR), and the USGS, Minnesota District, using a GIS.

Lake and marsh boundaries were overlaid on the subbasin boundaries to associate each lake and marsh with a subbasin. The total area of lakes and marshes within each subbasin was calculated by the GIS. Total marsh area plus total lake area is defined as storage area.

Main channels were delineated for each subbasin on the 7-1/2 minute topographic maps starting at the outflow of the subbasin and continuing upstream. Whenever the main channel joined with another stream, the stream upstream of the junction that drained the largest area was selected as the main channel. The main channel, which represents the watercourse that drains the greatest area, is continuous and is defined as a single trace that passes through marshes, lakes, and midline of rivers and braided streams from the basin outlet to an endpoint in the basin, generally at the basin divide. The main channels were digitized by the Minnesota 
Department of Transportation, using a computer aided drafting system and transferred to the GIS. Stream extensions that represent a portion of the main channel from the end of the mapped stream (blue line on 7-1/2 minute topographic maps) to an endpoint within the basin, generally at the basin divide, were digitized by USGS, Minnesota District, using a GIS. The main-channel data were overlaid onto the subbasin data to associate each main channel with its subbasin.

Elevation points were digitized at the intersection of topographic contour lines and main channels. The elevation data were digitized using a GIS. The elevation data was overlaid onto the main channel data to associate each elevation data point with a main channel. Two points on the main-channel, at 10 percent and at 85 percent of the main channel length from the basin outlet to the drainage divide, were located by the GIS. The elevations of these two points were interpolated from the digitized elevation data. Mainchannel slope was calculated by dividing the difference in elevation between these points by the distance along the stream channel between these points.

\section{Physical Characteristics of Cannon River Subbasins}

Physical characteristics determined for each of the subbasins shown on plate 1 are presented in table 1 . Subbasins are presented in order from headwaters to mouth. The rank of the subbasin stream is shown by indentation; whenever two subbasin streams joined, the stream draining the least cumulative area was assigned a lower rank and indented in the table.

The data for drainage area, and main-channel length are reported using three significant figures or rounded to the nearest one-hundredth of a unit. The data for lake area and storage area are rounded to the nearest one-tenth of a percent. The data for main-channel slope is reported to the nearest one-tenth of a foot per mile.

The following is an explanation of the terms used in table 1 and plate 1:

Subbasin number. A seven digit number based on the Minnesota Common Stream and Watershed Numbering System (Minnesota Department of Natural Resources, 1981). The first two digits are 39 and identify the Cannon River Basin. The following three digits are arbitrary and were assigned by the DNR. The last two digits were added by the USGS, Minnesota District,, to identify additional subdivisions to the DNR's watersheds at locations of USGS gaging stations and to identify noncontributing areas.

Stream name. The name of the stream or ditch shown on 7-1/2 minute topographic maps. The relative position of the subbasin above other subbasins, streams, and gaging stations.
Outlet location. The U.S. Public Lands Survey System is used to describe the location where the stream exits the subbasin, down to quarter-quarter section. The description includes quarter-quarter section, section, township, and range.

Drainage area. That area, measured on a horizontal plane, enclosed by a topographic divide, within which direct surface runoff from precipitation normally flows by gravity into a watercourse above a specific point. This may include closed basins and other areas that do not contribute directly to surface runoff.

Lake area. The percentage of the drainage area labeled lacustrine (lakes) on U.S. Fish and Wildlife Service National Wetlands Inventory Data.

$\underline{\text { Storage area. }}$ The percentage of a drainage area labeled lacustrine (lakes) and palustrine (marsh) on U.S. Fish and Wildlife Service National Wetlands Inventory Data. Marsh areas shown on plate 1 are from USGS 1:100,000 Digital Ling Graph data 1993.

Main-channel length. The total length of the main channel from the basin outlet to a point within the basin (generally at the basin divide) representing the watercourse that drains the greatest area.

Main-channel slope. The average slope of the watercourse between the points at 10 and at 85 percent of the distance along the main channel from the basin outlet to the drainage divide.

Stream extension. A representation of the main channel from the end of the mapped stream line (blue line on 7-1/2 minute topographic maps) to an endpoint within the basin, generally at the basin divide. This is done by interpreting topographic relief so that the extension of the main channel represents the watercourse draining the greatest area.

\section{References Cited}

Minnesota Department of Natural Resources, 1981, The common stream and watershed numbering system: Minnesota Department of Natural Resources Stream Inventory and Data Retrieval Systems Report 7002, unpaged.

Thompson, M.M., 1987, Maps for America, 3d edition: U.S. Geological Survey, 265 p.

U.S. Geological Survey, 1974, Hydrologic unit map--1974 State of Minnesota: 1 plate, scale 1:500,000.

U.S. Fish \& Wildlife Service, National Wetlands Inventory Digital Data: Oct. 1981 to present 
Table 1.-Physical characteristic data for the Cannon River Basin.

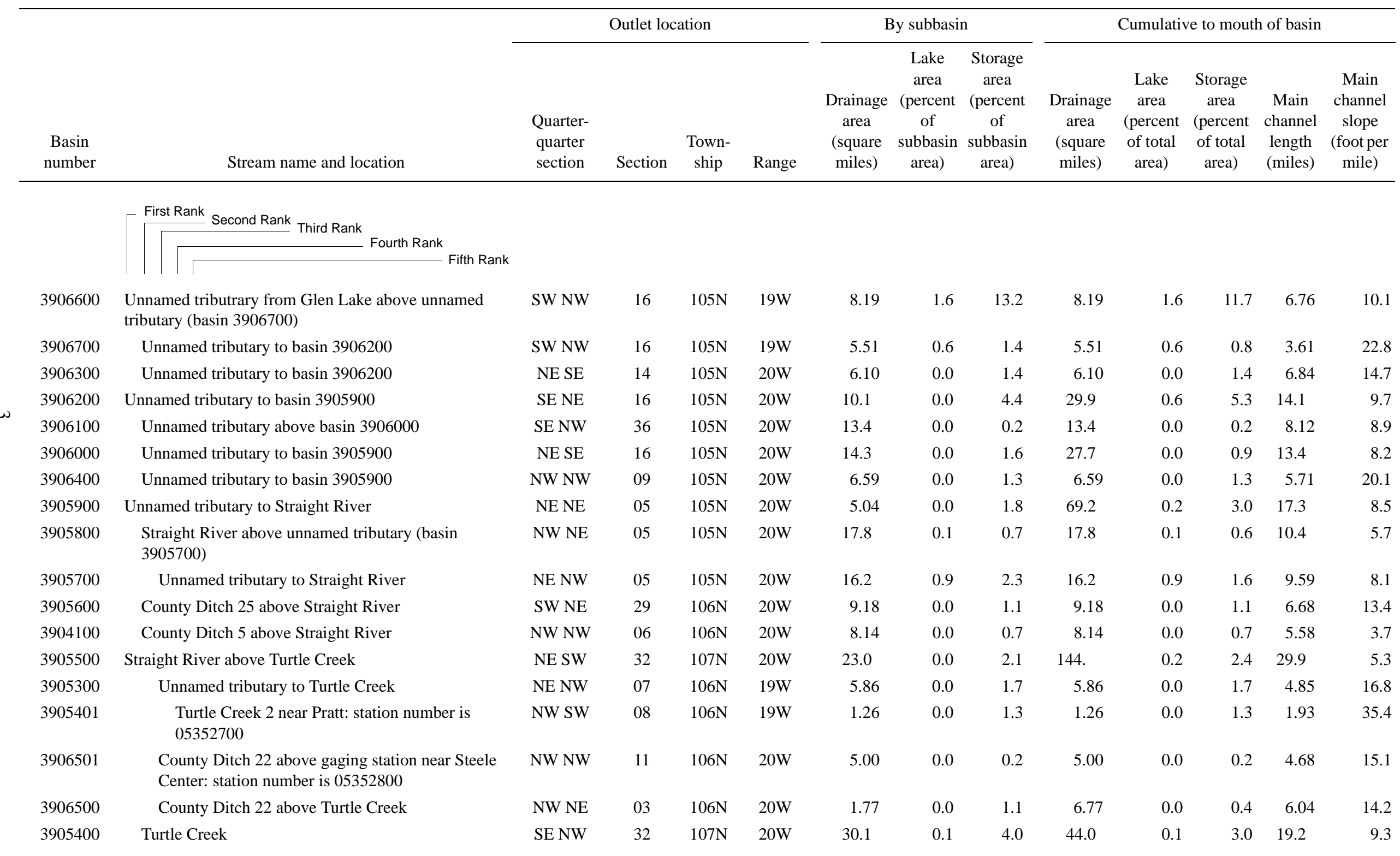


Table 1.-Physical characteristic data for the Cannon River Basin-Continued.

\begin{tabular}{|c|c|c|c|c|c|c|c|c|c|c|c|c|c|}
\hline \multirow[b]{2}{*}{$\begin{array}{l}\text { Basin } \\
\text { number }\end{array}$} & \multirow[b]{2}{*}{ Stream name and location } & \multicolumn{4}{|c|}{ Outlet location } & \multicolumn{3}{|c|}{ By subbasin } & \multicolumn{5}{|c|}{ Cumulative to mouth of basin } \\
\hline & & $\begin{array}{l}\text { Quarter- } \\
\text { quarter } \\
\text { section }\end{array}$ & Section & $\begin{array}{l}\text { Town- } \\
\text { ship }\end{array}$ & Range & $\begin{array}{l}\text { Drainage } \\
\text { area } \\
\text { (square } \\
\text { miles) }\end{array}$ & $\begin{array}{c}\text { Lake } \\
\text { area } \\
\text { (percent } \\
\text { of } \\
\text { subbasin } \\
\text { area) }\end{array}$ & $\begin{array}{c}\text { Storage } \\
\text { area } \\
\text { (percent } \\
\text { of } \\
\text { subbasin } \\
\text { area) }\end{array}$ & $\begin{array}{l}\text { Drainage } \\
\text { area } \\
\text { (square } \\
\text { miles) }\end{array}$ & $\begin{array}{l}\text { Lake } \\
\text { area } \\
\text { (percent } \\
\text { of total } \\
\text { area) }\end{array}$ & $\begin{array}{l}\text { Storage } \\
\text { area } \\
\text { (percent } \\
\text { of total } \\
\text { area) }\end{array}$ & $\begin{array}{l}\text { Main } \\
\text { channel } \\
\text { length } \\
\text { (miles) }\end{array}$ & $\begin{array}{l}\text { Main } \\
\text { channel } \\
\text { slope } \\
\text { (foot per } \\
\text { mile) }\end{array}$ \\
\hline 3904200 & Straight River above Maple Creek & NE NE & 09 & $107 \mathrm{~N}$ & $20 \mathrm{~W}$ & 16.5 & 0.3 & 3.3 & 204. & 0.2 & 2.7 & 38.8 & 4.0 \\
\hline 3905200 & Unnamed tributary to Maple Creek & NW NW & 09 & $107 \mathrm{~N}$ & $19 \mathrm{~W}$ & 16.3 & 0.0 & 1.6 & 16.3 & 0.0 & 1.6 & 8.61 & 7.9 \\
\hline 3905100 & $\begin{array}{l}\text { Maple Creek above unnamed tributary (basin } \\
\text { 3905200) }\end{array}$ & NW NW & 09 & $107 \mathrm{~N}$ & $19 \mathrm{~W}$ & 6.06 & 0.0 & 12.5 & 6.06 & 0.0 & 12.5 & 4.52 & 11.2 \\
\hline 3904300 & Maple Creek & NE NE & 09 & $107 \mathrm{~N}$ & $20 \mathrm{~W}$ & 16.1 & 0.0 & 2.7 & 38.5 & 0.0 & 3.8 & 17.1 & 6.5 \\
\hline 3903400 & Unnamed tributary to Rice Lake & SE SE & 04 & $107 \mathrm{~N}$ & $22 \mathrm{~W}$ & 5.20 & 22.6 & 31.4 & 5.20 & 22.6 & 10.0 & 3.86 & 6.3 \\
\hline 3903300 & Rice Lake outlet & NW SW & 03 & $107 \mathrm{~N}$ & $22 \mathrm{~W}$ & 14.0 & 1.9 & 14.8 & 19.2 & 7.5 & 13.2 & 6.61 & 5.7 \\
\hline 3903600 & Unnamed tributary to Goose Lake & SE NW & 14 & $107 N$ & $22 \mathrm{~W}$ & 6.66 & 0.0 & 8.6 & 6.66 & 0.0 & 8.6 & 5.19 & 2.0 \\
\hline 3903500 & Crane Creek above Judicial Ditch number 1 & NE NW & 01 & $107 \mathrm{~N}$ & $22 \mathrm{~W}$ & 9.50 & 7.4 & 22.7 & 35.3 & 6.1 & 14.3 & 9.35 & 3.9 \\
\hline 3903200 & Judicial Ditch number 1 to Crane Creek & SE SW & 36 & $108 \mathrm{~N}$ & $22 \mathrm{~W}$ & 7.80 & 0.0 & 17.7 & 7.80 & 0.0 & 17.7 & 5.43 & 6.0 \\
\hline 3903100 & Judicial Ditch number 1 to Crane Creek & SE SW & 32 & $108 \mathrm{~N}$ & $21 \mathrm{~W}$ & 14.2 & 0.0 & 4.4 & 14.2 & 0.0 & 4.4 & 4.91 & 2.5 \\
\hline 3904000 & County Ditch number 21 to basin 3903900 & SW SW & 19 & $107 \mathrm{~N}$ & $21 \mathrm{~W}$ & 10.7 & 0.0 & 0.8 & 10.7 & 0.0 & 0.8 & 7.29 & 8.8 \\
\hline 3903900 & Judicial Ditch number 1 to Crane Creek & SE SW & 05 & $107 \mathrm{~N}$ & $21 \mathrm{~W}$ & 8.77 & 0.0 & 0.5 & 19.5 & 0.0 & 0.7 & 11.6 & 5.9 \\
\hline 3903800 & Crane Creek & SE NE & 20 & $108 \mathrm{~N}$ & $20 \mathrm{~W}$ & 28.9 & 0.0 & 0.9 & 106. & 2.0 & 8.5 & 23.2 & 2.1 \\
\hline 3904500 & $\begin{array}{l}\text { Medford Creek above unnamed tributary (basin } \\
\text { 3904600) }\end{array}$ & SE SW & 14 & $108 \mathrm{~N}$ & $20 \mathrm{~W}$ & 7.53 & 0.0 & 1.1 & 7.53 & 0.0 & 1.1 & 6.38 & 19.4 \\
\hline 3904600 & Unnamed tributary to Medford Creek & SE SW & 14 & $108 \mathrm{~N}$ & $20 \mathrm{~W}$ & 7.25 & 0.0 & 1.2 & 7.25 & 0.0 & 1.2 & 7.57 & 17.2 \\
\hline 3904800 & Medford Creek & NW SE & 04 & $108 \mathrm{~N}$ & $20 \mathrm{~W}$ & 7.48 & 0.0 & 2.8 & 22.3 & 0.0 & 1.7 & 11.7 & 18.8 \\
\hline 3910200 & Straight River above Mud Creek & NW SW & 21 & $109 \mathrm{~N}$ & $20 \mathrm{~W}$ & 6.77 & 0.0 & 4.5 & 418. & 0.6 & 6.1 & 53.2 & 3.4 \\
\hline
\end{tabular}


Table 1.-Physical characteristic data for the Cannon River Basin-Continued.

\begin{tabular}{|c|c|c|c|c|c|c|c|c|c|c|c|c|c|}
\hline \multirow[b]{2}{*}{$\begin{array}{l}\text { Basin } \\
\text { number }\end{array}$} & \multirow[b]{2}{*}{ Stream name and location } & \multicolumn{4}{|c|}{ Outlet location } & \multicolumn{3}{|c|}{ By subbasin } & \multicolumn{5}{|c|}{ Cumulative to mouth of basin } \\
\hline & & $\begin{array}{l}\text { Quarter- } \\
\text { quarter } \\
\text { section }\end{array}$ & Section & $\begin{array}{l}\text { Town- } \\
\text { ship }\end{array}$ & Range & $\begin{array}{l}\text { Drainage } \\
\text { area } \\
\text { (square } \\
\text { miles) }\end{array}$ & $\begin{array}{c}\text { Lake } \\
\text { area } \\
\text { (percent } \\
\text { of } \\
\text { subbasin } \\
\text { area) }\end{array}$ & $\begin{array}{l}\text { Storage } \\
\text { area } \\
\text { (percent } \\
\text { of } \\
\text { subbasin } \\
\text { area) }\end{array}$ & $\begin{array}{c}\text { Drainage } \\
\text { area } \\
\text { (square } \\
\text { miles) }\end{array}$ & $\begin{array}{l}\text { Lake } \\
\text { area } \\
\text { (percent } \\
\text { of total } \\
\text { area) }\end{array}$ & $\begin{array}{l}\text { Storage } \\
\text { area } \\
\text { (percent } \\
\text { of total } \\
\text { area) }\end{array}$ & $\begin{array}{l}\text { Main } \\
\text { channel } \\
\text { length } \\
\text { (miles) }\end{array}$ & $\begin{array}{l}\text { Main } \\
\text { channel } \\
\text { slope } \\
\text { (foot per } \\
\text { mile) }\end{array}$ \\
\hline 3905000 & Mud Creek & NW SW & 21 & $109 N$ & $20 \mathrm{~W}$ & 14.3 & 0.0 & 3.1 & 14.3 & 0.0 & 3.1 & 10.5 & 7.4 \\
\hline 3910101 & $\begin{array}{l}\text { Straight River above gaging station near Fairbault: } \\
\text { station number is } 05353800\end{array}$ & NW SE & 09 & $109 \mathrm{~N}$ & $20 \mathrm{~W}$ & 3.65 & 0.0 & 0.6 & 435. & 0.6 & 6.0 & 55.6 & 3.3 \\
\hline 3910100 & Straight River above Falls Creek & SW SE & 33 & $110 \mathrm{~N}$ & $20 \mathrm{~W}$ & 4.65 & 0.0 & 4.4 & 440. & 0.6 & 6.0 & 58.4 & 3.3 \\
\hline 3908000 & Falls Creek & SE SW & 33 & $110 \mathrm{~N}$ & $20 \mathrm{~W}$ & 12.5 & 0.0 & 0.1 & 12.5 & 0.0 & 0.1 & 10.4 & 16.1 \\
\hline 3908400 & $\begin{array}{l}\text { Cannon River above unnamed tributary from Lake } \\
\text { Dora }\end{array}$ & SW SW & 11 & $110 \mathrm{~N}$ & $23 \mathrm{~W}$ & 29.2 & 8.4 & 26.9 & 29.2 & 8.4 & 21.0 & 17.1 & 2.2 \\
\hline 3908500 & Unnamed tributary from Lake Dora & NE SE & 10 & $110 \mathrm{~N}$ & $23 \mathrm{~W}$ & 20.4 & 5.4 & 15.8 & 20.4 & 5.4 & 11.5 & 9.73 & 2.6 \\
\hline 3909200 & Little Cannon River & NE NE & 30 & $110 \mathrm{~N}$ & $23 \mathrm{~W}$ & 14.5 & 1.3 & 13.2 & 14.5 & 1.3 & 12.1 & 8.79 & 7.9 \\
\hline 3910301 & $\begin{array}{l}\text { Cannon River above gaging station near Kilkenny: } \\
\text { station number is } 05348550\end{array}$ & NE NE & 31 & $110 \mathrm{~N}$ & $23 \mathrm{~W}$ & 23.6 & 8.1 & 21.9 & 87.8 & 6.5 & 19.9 & 30.5 & 2.0 \\
\hline 3910700 & Lake Jefferson outlet & SW NW & 05 & $109 N$ & $24 \mathrm{~W}$ & 16.5 & 19.0 & 28.4 & 16.5 & 19.0 & 12.6 & 8.97 & 3.9 \\
\hline 3910600 & German Lake outlet & NW NE & 04 & $109 N$ & $24 \mathrm{~W}$ & 7.48 & 17.7 & 32.4 & 23.9 & 18.6 & 15.5 & 10.5 & 3.0 \\
\hline 3910500 & County Ditch 59 & SE SE & 07 & $109 N$ & $23 \mathrm{~W}$ & 18.1 & 1.7 & 17.9 & 42.1 & 11.3 & 18.1 & 16.2 & 2.0 \\
\hline 3910300 & Cannon River above Tetonka Lake & NE NE & 19 & $109 \mathrm{~N}$ & $23 \mathrm{~W}$ & 12.2 & 0.0 & 11.2 & 142. & 7.3 & 24.1 & 37.7 & 2.2 \\
\hline 3903700 & Unnamed tributary to Tetonka Lake & NW NW & 28 & $109 \mathrm{~N}$ & $23 \mathrm{~W}$ & 12.2 & 16.4 & 22.8 & 12.2 & 16.4 & 8.4 & 10.4 & 6.6 \\
\hline 3910400 & Cannon River above outlet of Tetonka Lake & NW NE & 27 & $109 N$ & $23 \mathrm{~W}$ & 10.8 & 20.2 & 25.3 & 165. & 8.8 & 27.2 & 40.9 & 2.2 \\
\hline 3902800 & Waterville Creek & SE SE & 27 & $109 \mathrm{~N}$ & $23 \mathrm{~W}$ & 21.0 & 1.5 & 10.6 & 21.0 & 1.5 & 9.4 & 12.6 & 10.4 \\
\hline 3902700 & White Water Creek & SE NW & 26 & $109 N$ & $23 \mathrm{~W}$ & 15.8 & 1.6 & 12.1 & 36.8 & 1.5 & 10.3 & 13.2 & 10.7 \\
\hline 3909400 & Cannon River above outlet of Lower Sakatah Lake & SW SE & 17 & $109 \mathrm{~N}$ & $22 \mathrm{~W}$ & 8.03 & 23.5 & 28.2 & 217. & 8.1 & 29.3 & 45.5 & 1.9 \\
\hline
\end{tabular}


Table 1.-Physical characteristic data for the Cannon River Basin-Continued.

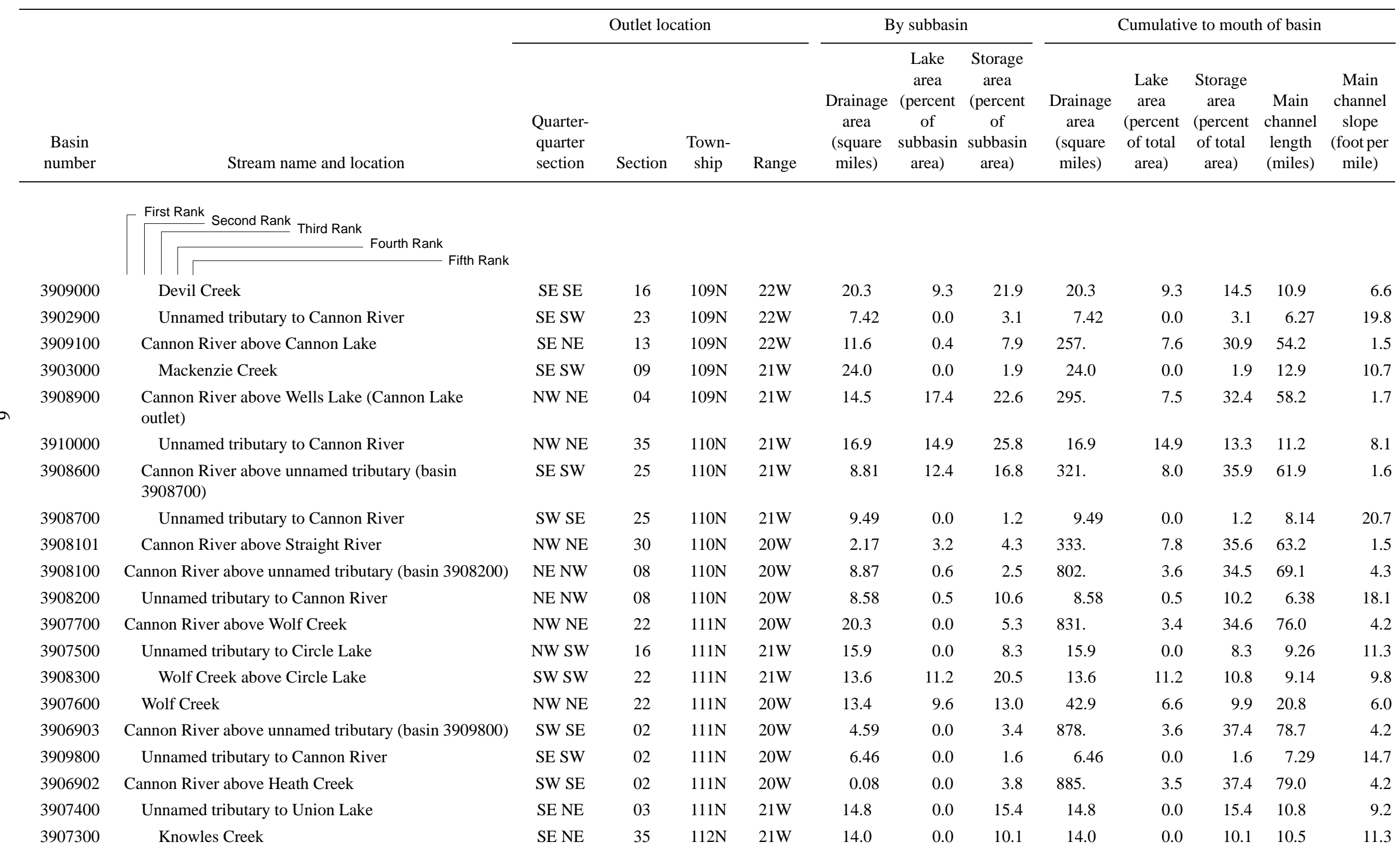


Table 1.-Physical characteristic data for the Cannon River Basin-Continued.

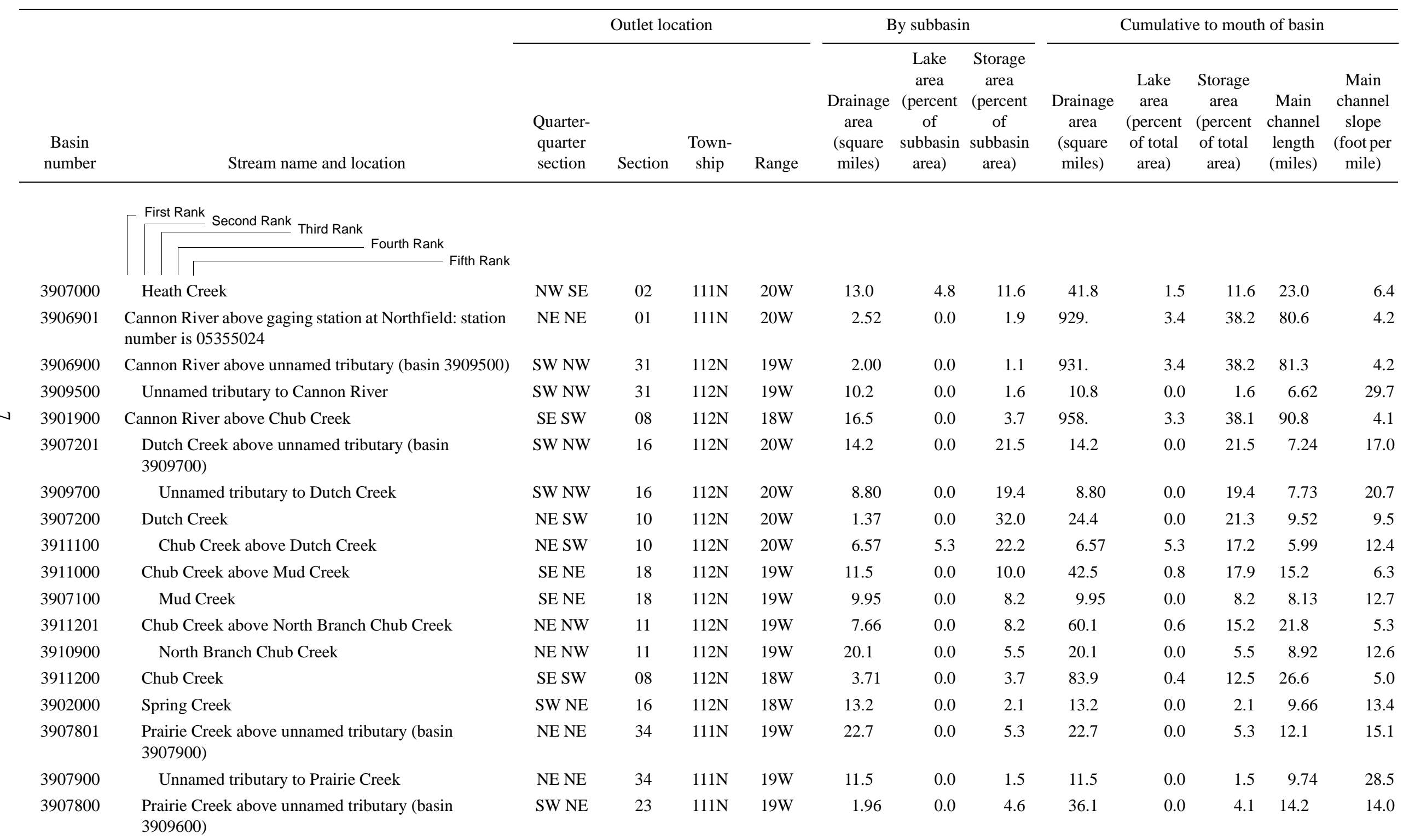


Table 1.-Physical characteristic data for the Cannon River Basin-Continued.

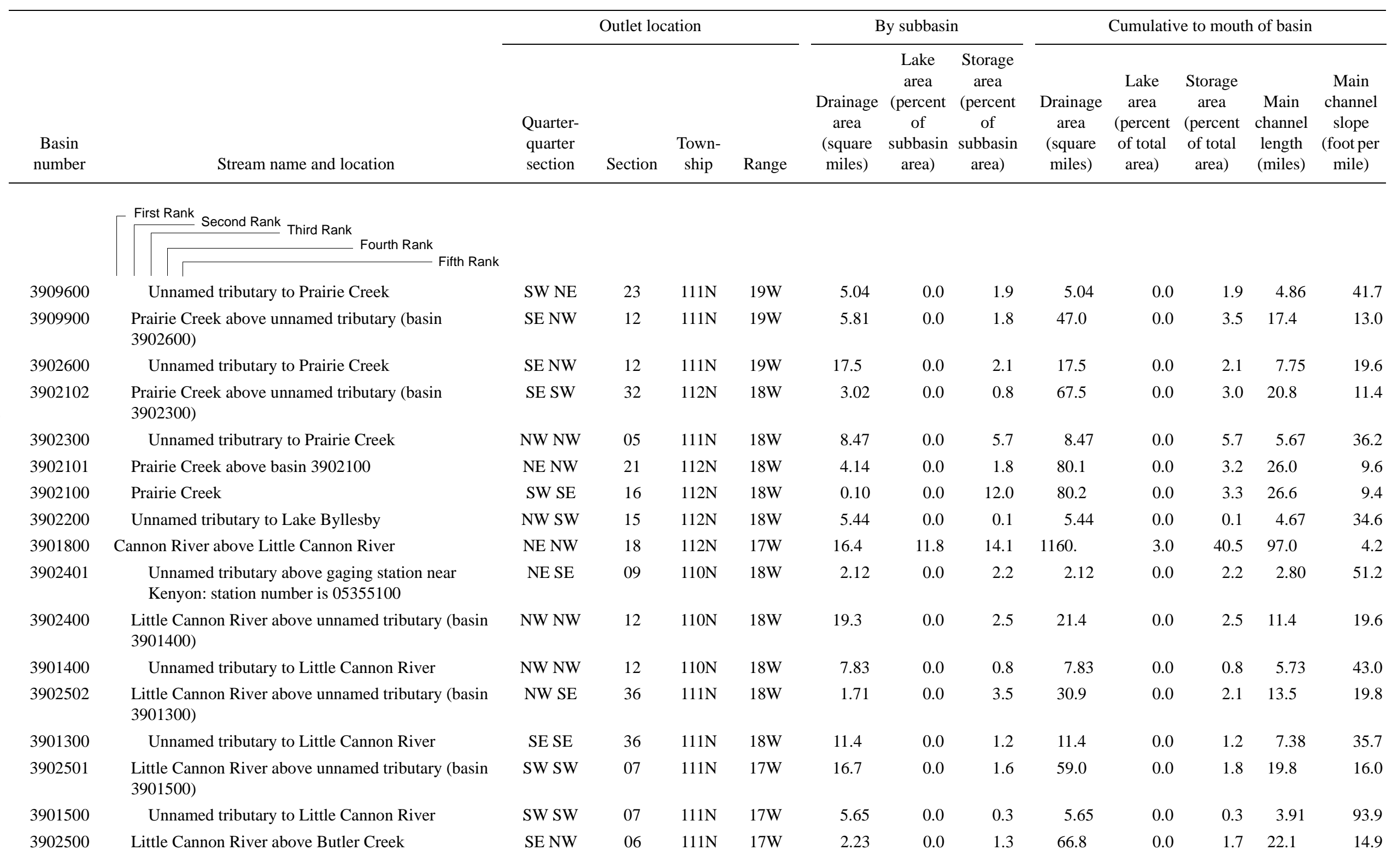


Table 1.-Physical characteristic data for the Cannon River Basin-Continued.

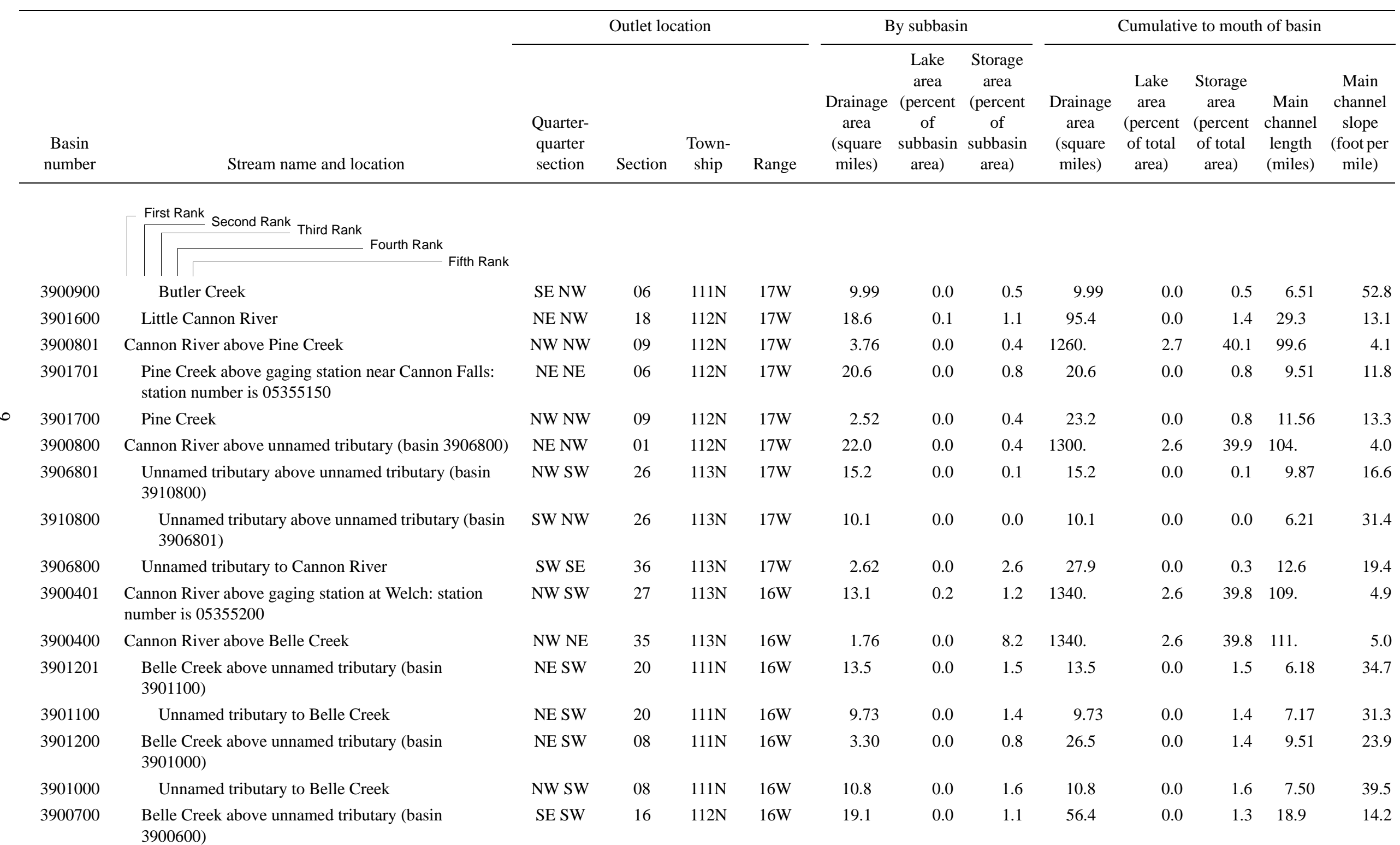


Table 1.-Physical characteristic data for the Cannon River Basin-Continued.

\begin{tabular}{|c|c|c|c|c|c|c|c|c|c|c|c|c|c|}
\hline \multirow[b]{2}{*}{$\begin{array}{l}\text { Basin } \\
\text { number }\end{array}$} & \multirow[b]{2}{*}{ Stream name and location } & \multicolumn{4}{|c|}{ Outlet location } & \multicolumn{3}{|c|}{ By subbasin } & \multicolumn{5}{|c|}{ Cumulative to mouth of basin } \\
\hline & & $\begin{array}{l}\text { Quarter- } \\
\text { quarter } \\
\text { section }\end{array}$ & Section & $\begin{array}{l}\text { Town- } \\
\text { ship }\end{array}$ & Range & $\begin{array}{c}\text { Drainage } \\
\text { area } \\
\text { (square } \\
\text { miles) }\end{array}$ & $\begin{array}{c}\text { Lake } \\
\text { area } \\
\text { (percent } \\
\text { of } \\
\text { subbasin } \\
\text { area) }\end{array}$ & $\begin{array}{c}\text { Storage } \\
\text { area } \\
\text { (percent } \\
\text { of } \\
\text { subbasin } \\
\text { area) }\end{array}$ & $\begin{array}{l}\text { Drainage } \\
\text { area } \\
\text { (square } \\
\text { miles) }\end{array}$ & $\begin{array}{c}\text { Lake } \\
\text { area } \\
\text { (percent } \\
\text { of total } \\
\text { area) }\end{array}$ & $\begin{array}{c}\text { Storage } \\
\text { area } \\
\text { (percent } \\
\text { of total } \\
\text { area) }\end{array}$ & $\begin{array}{l}\text { Main } \\
\text { channel } \\
\text { length } \\
\text { (miles) }\end{array}$ & $\begin{array}{l}\text { Main } \\
\text { channel } \\
\text { slope } \\
\text { (foot per } \\
\text { mile) }\end{array}$ \\
\hline & $\lceil$ First Rank Second Rank Third Rank & & & & & & & & & & & & \\
\hline 3900600 & Unnamed tributary to Belle Creek & NE NW & 21 & $112 \mathrm{~N}$ & $16 \mathrm{~W}$ & 13.4 & 0.0 & 0.7 & 13.4 & 0.0 & 0.7 & 10.1 & 29.1 \\
\hline 3900500 & Belle Creek & NW NE & 35 & $113 \mathrm{~N}$ & $16 \mathrm{~W}$ & 8.92 & 0.0 & 5.4 & 78.7 & 0.0 & 1.7 & 26.7 & 15.7 \\
\hline 3900101 & Cannon River above unnamed tributary (basin 3900200) & SE SW & 19 & $113 \mathrm{~N}$ & $15 \mathrm{~W}$ & 5.02 & 0.0 & 11.8 & 1430. & 2.4 & 39.6 & 114. & 5.0 \\
\hline 3900201 & $\begin{array}{l}\text { Unnamed tributary above gaging station near } \\
\text { Welch: station number is } 05355230\end{array}$ & NW NW & 14 & $113 N$ & $16 \mathrm{~W}$ & 0.08 & 0.0 & 0.0 & 0.08 & 0.0 & 0.0 & 0.22 & 164.9 \\
\hline 3900200 & Unnamed tributary to Cannon River & SE SW & 19 & $113 \mathrm{~N}$ & $15 \mathrm{~W}$ & 7.30 & 0.0 & 0.4 & 7.38 & 0.0 & 0.4 & 7.99 & 44.5 \\
\hline 3900100 & Cannon River & NE NE & 15 & $113 \mathrm{~N}$ & $15 \mathrm{~W}$ & 9.23 & 2.9 & 43.4 & 1440. & 2.4 & 40.1 & 120. & 5.1 \\
\hline 3900300 & Spring Creek & NW NE & 24 & $113 \mathrm{~N}$ & $16 \mathrm{~W}$ & 27.1 & 0.7 & 8.25 & 27.1 & 0.7 & 8.3 & 17.5 & 24.2 \\
\hline
\end{tabular}

